\title{
Pot Culture Studies on Integrated Effect of Bio agents, Organic Amendments, Nanoparticles and Chemicals on Crossandra (Crossandra infundibuliformis $\mathbf{L}$. Nees) Wilt Incited by Fusarium incarnatum (Desm.) Sacc
}

\author{
B. Mallaiah*, M. Muthamilan and D. Bhadru \\ Department of Plant Pathology, Maize Research Centre, ARI, \\ Rajendranagar, PJTSAU, Hyderabad, India \\ *Corresponding author
}

\section{A B S T R A C T}

\begin{tabular}{l} 
Ke y w or d s \\
Crossandra, \\
$\begin{array}{l}\text { Fusarium } \\
\text { incarnatum, } \\
\text { Organic } \\
\text { amendments, Nano } \\
\text { particles, Wilt }\end{array}$ \\
\hline Article Info \\
$\begin{array}{l}\text { Accepted: } \\
\text { 20 July 2020 } \\
\text { Available Online: } \\
\text { 10 August 2020 }\end{array}$ \\
\hline
\end{tabular}

\begin{abstract}
In pot culture studies with use selected bio agent, organic amendments, nanoparticle and chemical and their combinations tested, the treatment of $\mathrm{T}_{16}$ (Soil application(SA) of T.viride @ $2.5 \mathrm{~kg} / \mathrm{ha}$ at $20 \mathrm{DAP}+$ Module C(Soil drenching (SD) of carbendazim @ $0.1 \%$ at $30 \mathrm{DAP}+\mathrm{SA}$ of T.v (Tv-9) @ $2.5 \mathrm{~kg} / \mathrm{ha}$ at $50 \mathrm{DAP}+$ Foliar application ( FA ) of $P . f$ (Pf-18)@ 1.0 kg/ha at 70 DAP + FA of B.s (Bs-10) @ $1.0 / \mathrm{ha}$ at 90 DAP) recorded less per cent disease incidence $(2.8 \%)$ which accounted for 96.5 per cent disease reduction over control and increased flower yield of 38.6 per cent. $\mathrm{T}_{20}$ was the best treatment and recorded 0.5/5 lowest lesion index, which accounted for 88.9 per cent reduction of lesion index over control followed by $\mathrm{T}_{6} \mathrm{~T}_{16}$ and $\mathrm{T}_{9}$ were found to record maximum shoot length of $67.1 \mathrm{~cm}$ and $66.5 \mathrm{~cm}$ respectively which accounts for 58.6 and 58.1 per cent increased shoot length over control in addition to increased root length. The treatment $\mathrm{T}_{16}$ was found to record maximum mean reduction of pathogen population of $2.0 \times 10^{-3} \mathrm{cfu} / \mathrm{g}$ which accounted for 87.8 per cent over control and nematode population by 83.6 percent.
\end{abstract}

\section{Introduction}

Crossandra (Fire cracker) is an important commercial flower, mainly grown in India, Tropical Africa and Madagascar (Bailey, 1963). It is an erect, evergreen sub shrub growing to 1 meter with glossy, wavymargined leaves and fan-shaped flowers, which may appear at any time throughout the year and easy to grow. It can be cultivated even by small farmers. The flowers are commonly used for hair adornment. Though not fragrant, these flowers are very popular because of their attractive bright colour, light weight and good keeping quality. The flowers are used for making garlands, either alone or in combination with jasmine flowers. The estimated area under crossandra cultivation in India is around 1700 ha with a production of 430 tonnes (Venkatesan et al., 2004).).

Crossandra (Crossandra infundibuliformis) is affected by various fungal, bacterial and viral diseases. Among the various fungal diseases 
wilt disease caused by Fusarium spp. is one of the major problem in Crossandra production and limits the crop cultivation. Fusarium wilt of crossandra is a soil born disease and causes crop losses up to 92 percent. Some reports are also available stating that there is a consistent association of Fuarium spp. and nematode complex in crossandra wilt that are causing major crop yield losses (Srinivasan and Muthukrishnan, 1975). Till date systemic work was not carried to find the extend of losses and management of the crossandra wilt disease.

Management of this disease through chemicals and by the use of resistant varieties are possible to some extent. But the hazardous impact of agrochemicals on the environment, development of resistant mutants, escalating cost of pesticides and frequent breakdown of resistant varieties strongly demand a sustainable and an alternative management approach to disease control.

Use of microbes and organic amendments for disease management in agriculture is one of the most effective and eco-friendly disease control strategy. Nanoscale materials have emerged as novel antimicrobial agents owing to their high surface area to volume ratio and the unique chemical and physical properties, which increases their contact with microbes and their ability to penetrate cells. Also, nanotechnology has amplified the effectiveness of silver particles as antimicrobial agents. Kabir lamsal et al., (2011) used silver nanoparticles for effective controlling of anthracnose (Colletotrichum $s p \mathrm{p}$ ) in pepper and Powdery mildew (Sphaero thecafusca) in cucumber. Many workers successfully managed the plant diseases with use of bio control agents, organic amendments, nanoparticles and chemical fungicides in most efficient and eco-friendly way without effecting environment. So keeping all the factors in view, attempts were made to assess the effect of selected bio agent, organic amendments, nanoparticle and chemical on occurrence of crossandra wilt incited by $F$. incarnatum in pot culture.

\section{Materials and Methods}

All the pot culture experiments were conducted at the Department of Plant Pathology, Agricultural College and Research Institute, TNAU, Madurai. The local crossandra cultivar growing in the area was used in the experiment. The pathogen $(F$. incarnatum) mass multiplied on sand maize medium was incorporated in the pots at 3 per cent (w/w) and nematode was inoculated @ 1 nematode per gram of soil.

The native bio agents are collected from different places and screened for their antagonistic bactivity by following dual culture technique. The best antagonists, bacteria (Pseudomonas fluorescens (Pf-18), Bacillus subtilis (Bs-1), fungus, (Trichoderma viride (Tv-9)) were multiplied and formulated on talc powder and applied in pots @ $2.5 \mathrm{~kg} / \mathrm{ha}$. Different organic amendments are tested in laboratory by following poison food technique and the best organic amendment(Neem cake) showing highest inhibition of pathogen was used in pot culture studies@250 kg/ha.. Silver nanoparticles were synthesized by the reduction of silver nitrate in the presence of plant leaf extract of Tridax procumbense and tested for their antifungal activity in laboratory. The effective dose of Silver nanoparticles @ 800ppm was used in pot culture experiment.

The observations on the per cent disease incidence, growth parameters such as flower yield are recorded. Each treatment was replicated six times with each replication containing three pots with two plants in each pot. 


\section{Results and Discussion}

Among the twenty two treatments with different combinations tested, the treatment of $\mathrm{T}_{16}$ (SA of $T v @ 2.5 \mathrm{~kg} / \mathrm{ha}$ at $20 \mathrm{DAP}+$ Module C) recorded less per cent disease incidence $(2.8 \%)$ which accounted for 96.5 per cent disease reduction over control and it was followed by $T_{2}, T_{9}, T_{19}$ and $T_{20}$ with 5.5 per cent wilt incidence over control which accounted for 92.9 per cent reduction of wilt incidence over control. The minimum per cent disease reduction was observed in $\mathrm{T}_{4}$ (SA of neem cake@250 kg/ha + Module A) with 16.6 per cent wilt incidence which accounted for 78.7 per cent disease reduction over control. The observations on integrated treatmental effect on root lesion index indicates, $T_{20}$ was the best treatment which recorded 0.5/5 lesion index, which accounted for 88.9 per cent reduction of lesion index over control followed by $\mathrm{T}_{6}$ with root lesion index of $0.8 / 5$ which accounted for 81.6 per cent reduction of lesion index over control. The noted observation is that, in both the treatments Phorate 10G @ 10kg/ha was applied as soil application at 20 DAP (Table $1)$.

The results revealed that the integrated treatments with the biocontrol agents, oil cakes, nanoparticles and chemicals influenced the crossandra plant growth parameters. The treatment T16 (SAof Tv @ $2.5 \mathrm{~kg} / \mathrm{ha}$ at 20 DAP + Module C) and T9 (SA of Tv @ 2.5 $\mathrm{kg} / \mathrm{ha}$ at $20 \mathrm{DAP}+$ Module B) were found to record maximum shoot length of $67.1 \mathrm{~cm}$ and $66.5 \mathrm{~cm}$ respectively which accounts for 58.6 and 58.1 per cent increased shoot length over control. Both the treatments were statistically on par with each other. Minimum increased shoot length of 34.8 per cent was observed in T4. Whereas observations for influence of treatments on root length indicated, T16 and T20 were the best treatments with 32.7 and $32.4 \mathrm{~cm}$ root length which accounted for 46.6 and 45.3 per cent respectively increased growth over control and both these treatments were statistically on par with each other. Whereas minimum increase in root length was observed in T11. T16 (SA of Tv @ $2.5 \mathrm{~kg} / \mathrm{ha}$ at $20 \mathrm{DAP}+$ Module C) was found to record a maximum flower yield of 2515 $\mathrm{Kg} / \mathrm{ha}$, which accounted for 38.6 per cent increasd in flower yield over control followed by T20 (SA of Phorate @ $10 \mathrm{~kg} / \mathrm{ha}$ at 20 DAP + Module C) and T19 (SD of carbendazim @ $0.1 \%$ at $20 \mathrm{DAP})$ with increased flower yield of 36.2 and 35.1 per cent respectively over control. A minimum of 24.2 per cent of flower yield was increased in T11 when compared to control (Table 2).

The other observations on experimental results revealed that among the twenty two treatments with different integrations tested to study their effect on population density of P.delattrei, $\mathrm{T}_{20}$ (SA of Phorate 10G @ 10 $\mathrm{kg} / \mathrm{ha}$ at $20 \mathrm{DAP}$ plus Module C), $\mathrm{T}_{13}$ (SA of Phorate@10 kg/ha at 20 DAP + Module B), and $\mathrm{T}_{6}$ (SA of Phorate10G @ 10kg/ha at 20 DAP + Module A) were found to record highest reduction of nematode population at all the days tested with a mean reduction of 93.1, 92.8 and 91.5 per cent in nematode population respectively. $\mathrm{T}_{16}$ was found to be the next best treatment in reduction of nematode population, whereas minimum reduction of 68.2 per cent of nematode population was observed in $\mathrm{T}_{15}$ (Table 3 ).

Among the effect twenty two treatments tested on the population density of $F$. incarnatum, the results revealed that $\mathrm{T}_{16}$ (SA of Tv@ $2.5 \mathrm{~kg} / \mathrm{ha}$ at $20 \mathrm{DAP}+$ Module C) and $\mathrm{T}_{19}$ (SD of carbendazim @ $0.1 \%$ at 20 DAP plus Module C) were found to record maximum mean reduction of pathogen population of $2.0 \times 10^{-3} \mathrm{cfu} / \mathrm{g}$ which accounted for 87.8 per cent over control. 
Table.1 Effect of integration of different combinations of biocontrol agents, organic amendments, nanoparticles and chemicals on the occurrence of wilt of Crossandra in pot culture experiment

\begin{tabular}{|c|c|c|c|c|c|}
\hline $\begin{array}{l}\text { T. } \\
\text { No. }\end{array}$ & Name of the treatment & $\begin{array}{c}\text { Root lesion } \\
\text { index* (1-5 } \\
\text { scale) }\end{array}$ & $\begin{array}{c}\text { Percent } \\
\text { decrease } \\
\text { over control }\end{array}$ & $\begin{array}{l}\text { Wilt incidence } \\
(\%)^{*}\end{array}$ & $\begin{array}{c}\text { Disease } \\
\text { reduction over } \\
\text { control }(\%)\end{array}$ \\
\hline $\mathbf{T}_{1}$ & SA of P.fluorescens (Pf-18) @ $2.5 \mathrm{~kg} / \mathrm{ha}$ at $20 \mathrm{DAP}+$ Module A & $2.5(9.10)^{* *}$ & 44.4 & $8.3(16.74) * *$ & 89.4 \\
\hline $\mathbf{T}_{2}$ & SA of T.viride (Tv-9) @ 2.5 kg/ha at 20 DAP + Module A & $2.2(8.47)$ & 51.8 & $5.5(13.60)$ & 92.9 \\
\hline $\mathbf{T}_{3}$ & SA of B.subtilis(Bs-10)@2.5 kg/ha at 20 DAP + Module A & $2.7(9.38)$ & 40.9 & $13.9(21.83)$ & 82.3 \\
\hline $\mathbf{T}_{4}$ & SA of Neem cake @ 250 kg/ha at 20 DAP + Module A & $1.5(7.04)$ & 66.7 & $16.6(24.04)$ & 78.7 \\
\hline $\mathbf{T}_{5}$ & SD of carbendazim @ $0.1 \%$ at 20 DAP + Module A & $2.3(8.77)$ & 48.2 & $8.3(16.74)$ & 89.4 \\
\hline $\mathbf{T}_{6}$ & SA of Phorate10G @10 kg/ha at 20DAP + Module A & $0.8(5.22)$ & 81.6 & $8.3(16.74)$ & 89.4 \\
\hline $\mathbf{T}_{7}$ & FA of nano particles @ 800 ppm at 20 DAP + Module A & $2.0(8.12)$ & 55.6 & $11.1(19.43)$ & 85.8 \\
\hline $\mathbf{T}_{8}$ & SA of P.f(Pf-18) @ $2.5 \mathrm{~kg} / \mathrm{ha}$ at $20 \mathrm{DAP}+$ Module B & $2.0(8.12)$ & 55.6 & $11.1(19.43)$ & 85.8 \\
\hline $\mathbf{T}_{9}$ & SA of T.v(Tv-9) @2.5 kg/ha at 20 DAP + Module B & $1.8(7.77)$ & 59.3 & $5.5(13.60)$ & 92.9 \\
\hline $\mathbf{T}_{10}$ & SA of B.s (Bs-10) @ $2.5 \mathrm{~kg} / \mathrm{ha}$ at 20 DAP + Module B & $2.5(9.10)$ & 44.4 & $11.1(19.43)$ & 85.8 \\
\hline $\mathbf{T}_{11}$ & SA of Neem cake @ $250 \mathrm{~kg} / \mathrm{ha}$ at 20 DAP + Module B & $1.5(7.04)$ & 66.7 & $16.6(24.64)$ & 78.7 \\
\hline $\mathbf{T}_{12}$ & SD of carbendazim @ $0.1 \%$ at 20 DAP + Module B & $2.5(9.10)$ & 44.4 & $8.3(16.74)$ & 89.4 \\
\hline $\mathbf{T}_{13}$ & SA of Phorate10G @10 kg/ha at 20 DAP + Module B & $0.7(4.66)$ & 85.3 & $11.1(19.43)$ & 85.8 \\
\hline $\mathbf{T}_{14}$ & FA of nano particles@800 ppm at 20 DAP + Module B & $2.2(8.46)$ & 51.8 & $13.9(21.83)$ & 82.3 \\
\hline $\mathbf{T}_{15}$ & SA of $P . f(\mathrm{Pf}-18) @ 2.5 \mathrm{~kg} / \mathrm{ha}$ at $20 \mathrm{DAP}+$ Module C & $2.5(9.10)$ & 44.4 & $8.3(16.74)$ & 89.4 \\
\hline $\mathbf{T}_{16}$ & SA of T.v (Tv-9) @ $2.5 \mathrm{~kg} / \mathrm{ha}$ at $20 \mathrm{DAP}+$ Module C & $2.2(8.46)$ & 52.0 & $2.8(9.57)$ & 96.5 \\
\hline $\mathbf{T}_{17}$ & SA of B.s (Bs-10)@ $2.5 \mathrm{~kg} / \mathrm{ha}$ at 20 DAP + Module C & $2.8((9.68)$ & 37.1 & $8.3(16.74)$ & 89.4 \\
\hline $\mathbf{T}_{18}$ & SA of Neem cake @ $250 \mathrm{~kg} / \mathrm{ha}$ at 20 DAP + Module C & $1.5(7.04)$ & 66.7 & $13.9(21.83)$ & 82.3 \\
\hline $\mathbf{T}_{19}$ & SD of carbendazim @ 0.1\% at 20 DAP + Module C & $2.5(9.10)$ & 44.4 & $5.5(13.60)$ & 92.9 \\
\hline $\mathbf{T}_{\mathbf{2 0}}$ & SA of Phorate10G @10 kg/ha at 20 DAP + Module C & $0.5(4.07)$ & 88.9 & $5.5(13.60)$ & 92.9 \\
\hline $\mathbf{T}_{21}$ & FA of nanoparticles @800 ppm at 20 DAP + Module C & $2.5(9.10)$ & 44.4 & $8.3(16.74)$ & 89.4 \\
\hline $\mathbf{T}_{22}$ & Inoculation of pathogen and nematode only & $4.5(12.24)$ & - & $78.2(62.23)$ & - \\
\hline \multicolumn{2}{|r|}{$\mathrm{CD}(\mathbf{P}=\mathbf{0 . 0 5})$} & 0.14 & - & 1.2 & - \\
\hline \multicolumn{2}{|r|}{$\mathbf{S E}(\mathbf{m}) \pm$} & 0.05 & - & 0.42 & - \\
\hline
\end{tabular}

* Mean of six replications

** Figure in the parentheses are arc sine transformed values

Module A.= Foliar application (FA) of nano particles@ @ 800ppm at 30 DAP + SA of T. viride(Tv-9)@ $2.5 \mathrm{~kg} / \mathrm{ha}$ at 50 DAP + FA of P.f(Pf-18)@ 1.0 kg/ha at 70 DAP + FA of ofB.s(Bs-10) @ $1.0 \mathrm{~kg} / \mathrm{ha}$ at $90 \mathrm{DAP}$

Module B = FA of carbendazim @ 0.1\% at 30 DAP + SA of T.v (Tv-9) @ $2.5 \mathrm{~kg} / \mathrm{ha}$ at $50 \mathrm{DAP}+\mathrm{FA}$ of $P . f(\mathrm{Pf}-18)$ @ $1.0 \mathrm{~kg} / \mathrm{ha}$ at $70 \mathrm{DAP}+\mathrm{FA}$ of $B . s @ 1.0 \mathrm{~kg} / \mathrm{ha}$ at $90 \mathrm{DAP}$

Module C= SD of carbendazim @ 0.1\% at 30 DAP + SA of T.v (Tv-9) @ 2.5 kg/ha at 50 DAP + FA of P.f(Pf-18)@ $1.0 \mathrm{~kg} / \mathrm{ha}$ at 70 DAP + FA of B.s (Bs-10) @ 1.0/ha at 90 DAP 
Table.2 Effect of integration of different combinations of biocontrol agents, organic amendments, nanoparticles and chemicals on the plant growth parameters of Crossandra in pot culture experiment

\begin{tabular}{|c|c|c|c|c|c|c|c|}
\hline \multirow{2}{*}{$\begin{array}{l}\text { T. } \\
\text { No. }\end{array}$} & \multirow[t]{2}{*}{ Name of the treatment } & \multicolumn{2}{|c|}{ Shoot } & \multicolumn{2}{|c|}{ Root } & \multirow{2}{*}{$\begin{array}{l}\text { Flower yield } \\
\text { Kg/ha*** }\end{array}$} & \multirow[b]{2}{*}{$\begin{array}{c}\text { Yield } \\
\text { increase } \\
\text { over } \\
\text { control } \\
(\%)\end{array}$} \\
\hline & & Length $(\mathrm{cm})^{*}$ & $\begin{array}{c}\text { Percent } \\
\text { increase } \\
\text { over } \\
\text { control }\end{array}$ & Length $(\mathrm{cm})^{*}$ & $\begin{array}{c}\text { Percent } \\
\text { increase } \\
\text { over } \\
\text { control }\end{array}$ & & \\
\hline $\mathbf{T}_{1}$ & $\begin{array}{l}\text { SA of P.fluorescens }(P f-18) @ 2.5 \mathrm{~kg} / \mathrm{ha} \text { at } 20 \mathrm{DAP}+\text { Module } \\
\text { A }\end{array}$ & $60.7(51.67)^{* *}$ & 46.6 & $29.5(32.88)^{* *}$ & 32.3 & $\begin{array}{c}2360 \\
(44.58)^{* *}\end{array}$ & 30.0 \\
\hline $\mathbf{T}_{2}$ & SA of T.viride (Tv-9) @ $2.5 \mathrm{~kg} / \mathrm{ha}$ at $20 \mathrm{DAP}+$ Module A & $63.3(52.71)$ & 52.9 & $31.6(34.19)$ & 41.7 & $2434(49.33)$ & 34.1 \\
\hline $\mathbf{T}_{3}$ & SA of B.subtilis (Bs-10) @ 2.5kg/ha at 20 DAP + Module A & $58.2(49.71)$ & 40.6 & $28.4(32.18)$ & 27.4 & $2339(48.36)$ & 28.9 \\
\hline $\mathbf{T}_{4}$ & SA of Neem cake@ 250 kg/ha at 20 DAP + Module A & $55.8(48.32)$ & 34.8 & $27.9(31.87)$ & 25.1 & $2264(47.58)$ & 24.8 \\
\hline $\mathbf{T}_{5}$ & SD of carbendazim @ 0.1\% at 20 DAP + Module A & $58.9(50.11)$ & 42.3 & $31.1(33.88)$ & 39.5 & $2359(48.57)$ & 30.0 \\
\hline $\mathbf{T}_{6}$ & SA of Phorate10G @10 kg/ha at 20DAP + Module A & $60.3(50.93)$ & 45.7 & $31.9(34.37)$ & 43.0 & $2401(49.00)$ & 32.3 \\
\hline $\mathbf{T}_{7}$ & FA of nano particles @ 800 ppm at 20 DAP + Module A & $60.3(50.93)$ & 45.7 & $30.6(33.57)$ & 37.2 & $2380(48.78)$ & 31.1 \\
\hline $\mathbf{T}_{8}$ & SA of $P . f(\mathrm{Pf}-18) @ 2.5 \mathrm{~kg} / \mathrm{ha}$ at $20 \mathrm{DAP}+$ Module B & $61.2(51.46)$ & 47.8 & $29.1(32.63)$ & 30.5 & $2359(48.57)$ & 30.0 \\
\hline $\mathbf{T}_{9}$ & SA of T.v (TV-9) @ $2.5 \mathrm{~kg} / \mathrm{ha}$ at 20 DAP + Module B & $66.5(54.13)$ & 58.1 & $29.9(33.13)$ & 34.1 & $2422(49.21)$ & 33.4 \\
\hline $\mathbf{T}_{10}$ & SA of B.s (Bs-10)@2.5 kg/ha at 20 DAP + Module B & $59.2(50.29)$ & 43.0 & $26.5(30.97)$ & 18.8 & $2338(48.35)$ & 28.8 \\
\hline $\mathbf{T}_{11}$ & SA of Neem cake @ $250 \mathrm{~kg} / \mathrm{ha}$ at 20 DAP + Module B & $56.4(48.66)$ & 36.2 & $25.8(30.51)$ & 15.7 & $2254(47.48)$ & 24.2 \\
\hline $\mathbf{T}_{13}$ & SA of Phorate10G @10 kg/ha at 20DAP + Module B & $59.7(50.58)$ & 44.2 & $31.4(34.06)$ & 40.8 & $2390(48.89)$ & 31.7 \\
\hline $\mathbf{T}_{14}$ & FA of nano particles@800 ppm at 20 DAP + Module B & $61.4(51.58)$ & 48.3 & $30.1(33.260$ & 35.0 & $2371(48.69)$ & 30.6 \\
\hline $\mathbf{T}_{15}$ & SA of $P . f(\mathrm{Pf}-18) @ 2.5 \mathrm{~kg} / \mathrm{ha}$ at $20 \mathrm{DAP}+$ Module C & $56.1(48.49)$ & 35.5 & $30.5(33.50)$ & 36.8 & $2380(48.78)$ & 31.1 \\
\hline $\mathbf{T}_{16}$ & SA of T.v (Tv-10) @ $2.5 \mathrm{~kg} / \mathrm{ha}$ at $20 \mathrm{DAP}+$ Module C & $67.1(53.99)$ & 58.6 & $32.7(34.86)$ & 46.6 & $2515(50.15)$ & 38.6 \\
\hline $\mathbf{T}_{17}$ & SA of B.s (Bs-10)@2.5 kg/ha at 20 DAP + Module C & $56.6(48.78)$ & 36.7 & $29.4(32.82)$ & 31.8 & $2358(48.56)$ & 29.9 \\
\hline $\mathbf{T}_{18}$ & SA of Neem cake @ $250 \mathrm{~kg} / \mathrm{ha}$ at 20 DAP + Module C & $54.2(47.39)$ & 30.9 & $28.6(32.31)$ & 28.3 & $2317(48.13)$ & 27.7 \\
\hline $\mathbf{T}_{19}$ & SD of carbendazim @ $0.1 \%$ at 20 DAP + Module C & $61.3(51.52)$ & 48.1 & $31.2(33.94)$ & 39.9 & $2452(49.52)$ & 35.1 \\
\hline $\mathbf{T}_{20}$ & SA of Phorate10G @10 kg/ha at 20 DAP + Module C & $59.8(50.46)$ & 44.4 & $32.4(34.68)$ & 45.3 & $2472(49.71)$ & 36.2 \\
\hline $\mathbf{T}_{21}$ & FA of nanoparticles@800 ppm at 20 DAP + Module C & $61.6(51.70)$ & 48.8 & $30.8(33.69)$ & 38.1 & $2401(49.00)$ & 32.3 \\
\hline $\mathbf{T}_{22}$ & Inoculation of pathogen and nematode only & $41.4(40.03)$ & - & $22.3(28.16)$ & - & $1815(42.60)$ & - \\
\hline \multicolumn{2}{|r|}{ CD $(P=0.05)$} & 3.36 & - & 1.68 & - & 7.60 & - \\
\hline \multicolumn{2}{|r|}{$\mathrm{SE}(\mathbf{m}) \pm$} & 1.20 & - & 0.59 & - & 2.70 & - \\
\hline
\end{tabular}

* Mean of three replication

** Figure in the parentheses are arc sine transformed values

*** Figure in the parentheses are square root transformed values 
Table.3 Effect of integration of different combinations of bio control agents, organic amendments, nanoparticles and chemicals on the population density of Pratylenchus delattrei associated with wilt of crossandra in pot culture experiment

\begin{tabular}{|c|c|c|c|c|c|c|c|}
\hline \multirow{2}{*}{$\begin{array}{l}\text { T. } \\
\text { No. }\end{array}$} & \multirow[t]{2}{*}{ Name of the treatment } & \multicolumn{5}{|c|}{ Nematode population per $200 c^{*}$} & \multirow{2}{*}{$\begin{array}{c}\text { Population } \\
\text { reduction over } \\
\text { control }(\%)\end{array}$} \\
\hline & & $\begin{array}{c}\text { Initial } \\
\text { population }\end{array}$ & 10 DALA & 30 DALA & 50 DALA & $\begin{array}{c}\text { Mean of } \\
\text { population }\end{array}$ & \\
\hline $\mathbf{T}_{1}$ & $\begin{array}{l}\text { SA of P.fluorescens }(P f-18) @ 2.5 \mathrm{~kg} / \mathrm{ha} \text { at } 20 \mathrm{DAP}+ \\
\text { Module A }\end{array}$ & 200 & $164(12.84)$ & $111(10.58)$ & $51(7.21)^{* *}$ & $108(10.21)$ & 71.4 \\
\hline $\mathbf{T}_{2}$ & $\begin{array}{l}\text { SA of T.viride }(T v-9) @ 2.5 \mathrm{~kg} / \mathrm{ha} \text { at } 20 \text { DAP + Module } \\
\text { A }\end{array}$ & 200 & $124(11.18)$ & $85(9.27)$ & $43(6.63)$ & $84(9.03)$ & 77.7 \\
\hline $\mathbf{T}_{3}$ & $\begin{array}{l}\text { SA of B.subtilis(Bs-10) @ } 2.5 \mathrm{~kg} / \mathrm{ha} \text { at } 20 \text { DAP + Module } \\
\text { A }\end{array}$ & 200 & $149(12.25)$ & $102(10.15)$ & $45(6.78)$ & $99(9.73)$ & 73.7 \\
\hline $\mathbf{T}_{4}$ & SA of Neem cake @ 250 kg/ha at 20 DAP + Module A & 200 & $103(10.20)$ & $70(8.42)$ & $39(6.32)$ & $71(8.31)$ & 81.2 \\
\hline $\mathbf{T}_{5}$ & SD of carbendazim @ 0.1\% at 20 DAP + Module A & 200 & $116(10.81)$ & $79(8.94)$ & $38(6.24)$ & $77(8.66)$ & 79.6 \\
\hline $\mathbf{T}_{6}$ & SA of Phorate10G @10 kg/ha at 20DAP + Module A & 200 & $50(7.14)$ & $35(6.00)$ & $13(3.74)$ & $32(5.63)$ & 91.5 \\
\hline $\mathbf{T}_{7}$ & FA of nano particles@800 ppm at 20 DAP + Module A & 200 & $164(12.84)$ & $112(10.63)$ & $50(7.14)$ & $109(10.20)$ & 71.1 \\
\hline $\mathbf{T}_{8}$ & SA of $P . f(P f-18) @ 2.5 \mathrm{~kg} / \mathrm{ha}$ at $20 \mathrm{DAP}+$ Module B & 200 & $174(13.23)$ & $119(11.00)$ & $56(7.55)$ & $117(10.57)$ & 69.0 \\
\hline $\mathbf{T}_{9}$ & SA of $T . v(T v-9) @ 2.5 \mathrm{~kg} / \mathrm{ha}$ at 20 DAP + Module B & 200 & $105(10.29)$ & $72(8.54)$ & $36(6.08)$ & $71(8.31)$ & 81.2 \\
\hline $\mathbf{T}_{10}$ & SA of B.s (Bs-10)@2.5 kg/ha at 20 DAP + Module B & 200 & $162(12.76)$ & $112(10.63)$ & $46(6.85)$ & $107(10.08)$ & 71.6 \\
\hline $\mathbf{T}_{11}$ & SA of Neem cake @ $250 \mathrm{~kg} / \mathrm{ha}$ at 20 DAP + Module B & 200 & $116(10.81)$ & $79(8.94)$ & $43(6.63)$ & $79(8.79)$ & 79.0 \\
\hline $\mathbf{T}_{12}$ & SD of carbendazim @ 0.1\% at 20 DAP + Module B & 200 & $101(10.10)$ & $69(8.37)$ & $28(5.38)$ & $66(7.95)$ & 82.5 \\
\hline $\mathbf{T}_{13}$ & SA of Phorate10G @ 10 kg/ha at 20DAP + Module B & 200 & $42(6.56)$ & $29(5.48)$ & $11(3.47)$ & $27(5.17)$ & 92.8 \\
\hline $\mathbf{T}_{14}$ & FA of nano particles@800 ppm at 20 DAP + Module B & 200 & $126(11.27)$ & $86(9.33)$ & $51(7.21)$ & $88(9.27)$ & 76.7 \\
\hline $\mathbf{T}_{15}$ & SA of $P . f(P f-18) @ 2.5 \mathrm{~kg} / \mathrm{ha}$ at 20 DAP + Module C & 200 & $179(13.41)$ & $122(11.10)$ & $60(7.810$ & $120(10.77)$ & 68.2 \\
\hline $\mathbf{T}_{16}$ & SA of $T . v(T v-9) @ 2.5 \mathrm{~kg} / \mathrm{ha}$ at $20 \mathrm{DAP}+$ Module C & 200 & $90(9.54)$ & $62(7.94)$ & $33(5.83)$ & $62(7.77)$ & 83.6 \\
\hline $\mathbf{T}_{17}$ & SA of B.s(Bs-10)@2.5 kg/ha at 20 DAP + Module C & 200 & $118(10.91)$ & $81(9.05)$ & $38(6.24)$ & $79(8.73)$ & 79.0 \\
\hline $\mathbf{T}_{18}$ & SA of Neem cake@ $250 \mathrm{~kg} / \mathrm{ha}$ at 20 DAP + Module C & 200 & $105(10.29)$ & $72(8.54)$ & $30(5.57)$ & $69(8.13)$ & 81.7 \\
\hline $\mathbf{T}_{19}$ & SD of carbendazim @ $0.1 \%$ at 20 DAP + Module C & 200 & $105(10.29)$ & $73(8.60)$ & $29(5.48)$ & $69(8.13)$ & 81.7 \\
\hline $\mathbf{T}_{\mathbf{2 0}}$ & SA of Phorate10G @ 10kg/ha at 20DAP + Module C & 200 & $42(6.56)$ & $29(5.48)$ & $8(3.00)$ & $26(5.01)$ & 93.1 \\
\hline $\mathbf{T}_{21}$ & FA of nanoparticles @ 800 ppm at 20 DAP + Module C & 200 & $130(11.44)$ & $89(9.49)$ & $43(6.63)$ & $87(9.19)$ & 76.9 \\
\hline $\mathbf{T}_{22}$ & Inoculation of pathogen and nematode only & 200 & $323(17.99)$ & $385(19.64)$ & $424(20.61)$ & $377(19.41)$ & - \\
\hline \multicolumn{2}{|r|}{ CD $(P=0.05)$} & - & 7.87 & 6.59 & 5.58 & 6.68 & - \\
\hline \multicolumn{2}{|r|}{$\mathrm{SE}(\mathbf{m}) \pm$} & - & 2.805 & 2.349 & 1.99 & 2.38 & - \\
\hline
\end{tabular}

* Mean of six replications

**Figure in the parentheses are square root transformed values

DALA $=$ Days after last application

DAP $\quad=$ Days after planting 
Table.4 Effect of integration of different combinations of bio control agents, organic amendments, nanoparticles and chemicals on the population density of $\mathrm{F}$. incarnatum causing wilt of crossandrain vivo

\begin{tabular}{|c|c|c|c|c|c|c|c|}
\hline \multirow{2}{*}{$\begin{array}{l}\text { T. } \\
\text { No. }\end{array}$} & \multirow[t]{2}{*}{ Name of the treatment } & \multicolumn{5}{|c|}{ Fungal cfu/g soil 10-3* } & \multirow{2}{*}{$\begin{array}{l}\text { Population } \\
\text { reduction over } \\
\text { control }(\%)\end{array}$} \\
\hline & & 0 DALA & 10 DALA & 30 DALA & 50 DALA & $\begin{array}{c}\text { Mean } \\
\text { population }\end{array}$ & \\
\hline $\mathbf{T}_{1}$ & $\begin{array}{l}\text { SA of P.fluorescens }(P f-18) @ 2.5 \mathrm{~kg} / \mathrm{ha} \text { at } 20 \mathrm{DAP}+ \\
\text { Module A }\end{array}$ & $5.1(2.47)^{* *}$ & $1.7(1.64)$ & $1.2(1.48)$ & $1.0(1.42)$ & $2.25(1.75)$ & 86.3 \\
\hline $\mathbf{T}_{2}$ & SA of T.viride $(T v-9) @ 2.5 \mathrm{~kg} / \mathrm{ha}$ at $20 \mathrm{DAP}+$ Module A & $4.9(2.43)$ & $1.6(1.61)$ & $1.1(1.45)$ & $0.9(1.38)$ & $2.13(1.72)$ & 87.0 \\
\hline $\mathbf{T}_{3}$ & $\begin{array}{l}\text { SA of B.subtilis (Bs-10)@ } 2.5 \mathrm{~kg} / \mathrm{ha} \text { at } 20 \text { DAP + Module } \\
\text { A }\end{array}$ & $4.9(2.43)$ & $1.8(1.67)$ & $1.4(1.55)$ & $1.2(1.48)$ & $2.33(1.78)$ & 85.8 \\
\hline $\mathbf{T}_{4}$ & SA of Neem cake@250 kg/ha at 20 DAP + Module A & $4.8(2.41)$ & $1.9(1.70)$ & $1.5(1.58)$ & $1.3(1.52)$ & $2.38(1.80)$ & 85.5 \\
\hline $\mathbf{T}_{5}$ & SD of carbendazim @ $0.1 \%$ at 20 DAP + Module A & $5.0(2.45)$ & $1.4(1.55)$ & $1.1(1.45)$ & $0.9(1.38)$ & $2.10(1.71)$ & 87.2 \\
\hline $\mathbf{T}_{6}$ & SA of Phorate10G @10 kg/ha at 20DAP + Module A & $4.9(2.43)$ & $2.1(1.76)$ & $1.7(1.64)$ & $1.2(1.48)$ & $2.48(1.83)$ & 84.9 \\
\hline $\mathbf{T}_{7}$ & FA of nano particles @ 800 ppm at 20 DAP + Module A & $4.9(2.43)$ & $2.3(1.82)$ & $1.6(1.61)$ & $1.2(1.48)$ & $2.50(1.84)$ & 84.8 \\
\hline $\mathbf{T}_{8}$ & SA of $P . f(P f-18) @ 2.5 \mathrm{~kg} / \mathrm{ha}$ at $20 \mathrm{DAP}+$ Module B & $4.9(2.43)$ & $1.9(1.70)$ & $1.4(1.55)$ & $1.1(1.45)^{* *}$ & $2.33(1.78)$ & 85.8 \\
\hline $\mathbf{T}_{9}$ & SA of $T \cdot v(T v-9) @ 2.5 \mathrm{~kg} / \mathrm{ha}$ at 20 DAP + Module B & $5.0(2.45)$ & $1.8(1.67)$ & $1.2(1.48)$ & $1.0(1.42)$ & $2.25(1.76)$ & 86.3 \\
\hline $\mathbf{T}_{10}$ & SA of B.s (Bs-10)@2.5 kg/ha at 20 DAP + Module B & $5.2(2.49)$ & $1.9(1.70)$ & $1.5(1.58)$ & $1.2(1.48)$ & $2.45(1.81)$ & 85.1 \\
\hline $\mathbf{T}_{11}$ & SA of Neem cake @ 250 kg/ha at 20 DAP + Module B & $5.1(2.47)$ & $2.1(1.76)$ & $1.7(1.64)$ & $1.4(1.55)$ & $2.58(1.86)$ & 84.3 \\
\hline $\mathbf{T}_{12}$ & SD of carbendazim @ $0.1 \%$ at 20 DAP + Module B & $4.9(2.43)$ & $1.5(1.58)$ & $1.2(1.48)$ & $1.0(1.42)$ & $2.15(1.73)$ & 86.9 \\
\hline $\mathbf{T}_{13}$ & SA of Phorate10G @10 kg/ha at 20DAP + Module B & $4.8(2.41)$ & $2.3(1.82)$ & $1.8(1.67)$ & $1.3(1.52)$ & $2.60(1.86)$ & 84.5 \\
\hline $\mathbf{T}_{14}$ & FA of nano particles @ 800 ppm at 20 DAP + Module B & $4.7(2.39)$ & $2.4(1.84)$ & $1.9(1.70)$ & $1.4(1.55)$ & $2.60(1.86)$ & 84.1 \\
\hline $\mathbf{T}_{15}$ & SA of $P . f(P f-18) @ 2.5 \mathrm{~kg} / \mathrm{ha}$ at $20 \mathrm{DAP}+$ Module C & $4.8(2.41)$ & $1.6(1.61)$ & $1.1(1.45)$ & $0.9(1.38)$ & $2.10(1.71)$ & 87.2 \\
\hline $\mathbf{T}_{16}$ & SA of $T . v(T v-9) @ 2.5 \mathrm{~kg} / \mathrm{ha}$ at $20 \mathrm{DAP}+$ Module C & $4.9(2.43)$ & $1.3(1.52)$ & $1.0(1.42)$ & $0.8(1.34)$ & $2.00(1.68)$ & 87.8 \\
\hline $\mathbf{T}_{17}$ & SA of B.s (Bs-10)@2.5 kg/ha at 20 DAP + Module C & $5.0(2.45)$ & $1.7(1.64)$ & $1.3(1.52)$ & $1.1(1.45)$ & $2.28(1.77)$ & 86.1 \\
\hline $\mathbf{T}_{18}$ & SA of Neem cake @ 250 kg/ha at 20 DAP + Module C & $4.9(2.43)$ & $1.9(1.70)$ & $1.4(1.55)$ & $1.1(1.45)$ & $2.33(1.78)$ & 85.8 \\
\hline $\mathbf{T}_{19}$ & SD of carbendazim @ $0.1 \%$ at 20 DAP + Module C & $4.8(2.41)$ & $1.2(1.52)$ & $1.1(1.45)$ & $0.9(1.38)$ & $2.00(1.68)$ & 87.8 \\
\hline $\mathbf{T}_{20}$ & SA of Phorate10G @10 kg/ha at 20DAP + Module C & $4.7(2.39$ & $1.8(1.67)$ & $1.4(1.55)$ & $1.1(1.45)$ & $2.28(1.77)$ & 86.3 \\
\hline $\mathbf{T}_{21}$ & FA of nanoparticles @ 800 ppm at 20 DAP + Module C & $4.9(2.43)$ & $2.1(1.76)$ & $1.6(1.61)$ & $1.2(1.48)$ & $2.46(1.82)$ & 85.1 \\
\hline $\mathbf{T}_{22}$ & Inoculation of pathogen and nematode only & $5.1(2.47)$ & $18.2(4.38)$ & $20.4(4.63)$ & $21.7(6.76)$ & $16.35(4.55)$ & - \\
\hline \multicolumn{2}{|r|}{ CD $(P=0.05)$} & 0.22 & 0.20 & 0.22 & 0.23 & 0.22 & - \\
\hline \multicolumn{2}{|c|}{$\mathrm{SE}(\mathbf{m}) \pm$} & 0.08 & 0.07 & 0.08 & 0.08 & 0.08 & - \\
\hline \multicolumn{8}{|c|}{$\begin{array}{l}\text { * Mean of six replications, } \\
{ }^{* *} \text { Figure in the parentheses are square root transformed values }\end{array}$} \\
\hline
\end{tabular}


The treatment $\mathrm{T}_{14}$ was found to be the less effective in reducing the pathogen population by recording 84.1 per cent reduction over control (Table 4). The results reflected that application of integrated treatments were found to be more effective in suppressing the crossandra wilt caused by $F$. Incarnatum rather than individual application of antagonistic organisms, chemicals, organic amendments or nanoparticles in the two experiments conducted separately under glass house conditions. Integrated module (T16) involving application of $T$. viride (Tv-9)as basa application at 20 DAP plus module C (Module $\mathrm{C}=\mathrm{SD}$ of carbendazim @ $0.1 \%$ at 30 DAP + SA of T.v (Tv-9) @ 2.5kg/ha at 50 DAP + FA of P.f (Pf-18)@ $1.0 \mathrm{~kg} / \mathrm{ha}$ at 70 $\mathrm{DAP}+\mathrm{FA}$ of B.s(Bs-10) @ $1.0 \mathrm{~kg} / \mathrm{ha}$ at 90 DAP) was found to be the best treatment followed by T20 where phorate $10 \mathrm{G}$ was applied as basal application at 20 DAP $($ T20 = SA of Phorate10G @10 kg/ha at 20DAP + Module C).

Among the three kinds of modules tested, modules involving soil drenching of carbendazim as basal at 20 DAP and $T$. Viride at 50 DAP was found to be more efficient in disease reduction, and improved plant growth parameters and reduction of pathogen populations than foliar application of carbendazim or foliar application of silver nanoparticles at the same time interval. Silver nanoparticles alone as well as in combinations also offering good control either on par or better than with bio control agents

Several approaches have been used to include combined application of two or more biocontrol stains to enhance the level and consistency in disease control (Pierson and Weller, 1994). Saravanakumar (2002) reported that mixture of two PGPR strains was also found to be effective in reducing the disease incidence of root rot of green gram under glasshouse conditions. Similarly several literature have documented the use of biocontrol agents in combination was more effective for management of plant diseases and pathogens compared to individual agents (Saravanakumar et al., 2007; Young Cheol et al., 2008)

Van peer and Schippers (1988) reported that there was increase in the root length, shoot length of tomato, cucumber, lettuce and potato as a result of bacterization with Pseudomonas strains. The increase in plant growth might be associated with secretion of auxins, gibberellins and cytokinins (Dubeikovsky et al., 1993). Salah EddinKhabbaz (2006) reported that there was significant increase in seed germination, vigour index and dry weight with $P$. Fluorescens isolates ( $\mathrm{Pf} 1$ and MMP) under glass house conditions. Linderman (1989) indicated that the organic amendments acted through more than one mechanism either simultaneously or sequentially in the suppression of the disease. These were also known to influence soil physical characters such as pore size, aeration, temperature, water retention capacity which are essential for rapid extension of the root systems, better uptake of nutrients, retention of added nitrogen for longer period and finally for better plant vigour for resisting the pathogen attack. $T$. viride and T. harzianumwere proven to be compatible with $P$. flurorescens and suppressed seedling disease of tomato in greenhouse condition (Rini and Sulochana, 2007). The Trichoderma spp separately protected tomato seedlings against Fusarium wilt. Plants treated one week before inoculation with the pathogen appeared healthy and with no wilting symptoms in pot culture condition (Ali et al., 2009).

The maximum percentage of survival rates of tomato seedlings against $F$. Solani had attained in response to combined application of $T$. viride and B. subtilis rather than their 
individual application (Morsy et al., 2009). Carbendazim was highly effective in controlling wilt of pea upto 83 per cent (Sharma, 2011). In tomato, minimum wilt incidence of 14.9 per cent was recorded by seed priming with $T$. viride and soil application of neem cake (Barnwal et al., 2011). It has been established that Trichoderma spp. inhibit pathogenic invasion through the phenomenon of mycoparasitism, antibiosis and competition (Anwar et al., 2008). Najar et al., (2011) evaluated the native biocontrol agents against Fusarium solani f. sp. Melongenae causing wilt of brinjal and reported that $P$. fluorescens followed by T.viride were best in reducing the disease incidence of brinjal wilt.

The results were consistent with the findings of Vimala et al., (2009) and Thiruvudainambi et al., (2010) who reported the efficacy of $T$. viride and neem cake formulation against $S$. rolfsii, M.phaseolina, and F.oxysporum. Soilborne plant pathogens affecting agricultural plants can be controlled by the use of species of Trichoderma, B. subtilis, P. fluorescence (Anitha and Dass, 2011. Sumana et al., (2012) reported that soil drenching of carbendazim was highly effective in reducing tobacco wilt than foliar application.

\section{References}

Anitha, A., and M.A. Dass, 2011. Activation of rice plant growth against Rhizoctonia solani using Pseudomonas fluorescens, Trichoderma and salicylic acid. Research in Biotechnology. (2): 7-12.

Anwar, A., G.N. Bhat and K.A, Bhat, 2008. Mycoparasitic behaviour of certain bioagents against sheath blight pathogen (Rhizoctonia solani) of rice. J. Mycol Pl Pathol.,(38): 135-137.

Bailey, L.H., 1963. The standard cyclopedia of horticulture Newyork: The Mac Millan
Barnwal, M.K., D. Maiti and A.C. Pandey, 2011. Management of tomato wilt caused by Fusarium oxysporum f.sp. lycopersici by using bioagents and organic amendments. Indian Phytopathol., (64): 194-196.

Dubeikovsky, A.N., E.A. Mordukhova, V.V. Kochethov, F.Y. Polikarpova and A.M. Boronin, 1993. Growth promotion of black current soft wood cuttings by recombinant strain Pseudomonas fluorescens BSP53 synthesizing an increased amount of indole -3 -acetic acid. Soil Biol. Biochem., (25): 12771281.

Kabir Lamsal, Sang Woo Kim, JinHee Jung, Yun Seok Kim, KyoungSu Kim and YounSu Lee, 2011. Inhibition effect of silver nanoparticles against powdery mildews on Cucumber and Pumpkin. Mycobiology.,(39): 26-32

Linderman, R.G, 1989. Organic amendments and soil borne diseases. Canadian J. Pl. Pathol., 11: 180-183.

Morsy, E.B., Abdel-Kawi, K.A. and Khalil, M.N.A, 2009. Efficiency of Trichoderma viride and Bacillus subtilis as biocontrol agents against $F$.solani on tomato plants. Egypt. J. Phytopathol., (37): 47-57.

Pierson, E.A., and D.M. Weller, 1994. Use of mixture of fluorescent pseudomonads to suppress take-all and improve the growth of wheat. Phytopathology,(84): 940-947.

Rini, C.R. and Sulochana, K.K, 2007. Usefulness of Trichoderma and Pseudomonas against Rhizoctonia solani and Fusarium oxysporum infecting tomato. J. Trop. Agric., 45(1-2): 21-28.

Salah Eddin Khabbaz, 2006. Selection of plant growth promoting Rhizobacteria through molecular and biochemical approaches to manage root rot and bacterial blight of cotton. Ph.D.,(Ag.), Thesis. Tamil Nadu Agricultural 
University, Coimbatore. pp. 87-90.

Saravanakumar, D. 2002. Rhizobacteria induced systemic resistance against Biotroph (Exobasidium vexans) and Necrotroph (Macrophomina phaseolina) pathogens in tea and green gram. M.Sc., (Ag.) Thesis, Tamil Nadu Agricultural University, Coimbatore, India. Pp.120.

Sarvanakumar, D., Kannappan,M., Nallathambi, L and Sethuraman, S, 2007. Pseudomonos-induced defence molecules in rice plants against leaf foldr pest. Pest management science, (63): 714-721.

Sharma, P, 2011. Evaluation of disease control and plant growth promotion potential of biocontrol agents on Pisum sativum and comparison of their activity with popular chemical control agentcarbendazim. J. Toxicol. Environ. Health Sci.,3:

Srinivasan, A., T.S. Muthukrishnan.1975. A note on nematode fungal complex in crossandra (Crossandra undulae foliae Salisb) in Coimbatore. Current science,(20): 743-44127-138.

Sumana, K., Ramakrishnan, S., Sreenivas, S.S. and Devaki1, N.S. 2012. Field evaluation of promising fungicides and bioagents against Fusarium wilt and root knot complex disease in FCV tobacco crop. J.Agri.Technol., (8): 983-991.

Van Peer, R. and B. Schippers, 1988. Plant growth response in bacterization with selected Pseudomonas spp. strains and rhizosphere microbial development in hydrophonic cultures. Canadian J. Microbiol., 35: 456-463.

Venkatesan, S. Arumugam Shakila and M. Kadeer Mohideen, 2004. Effect of growth of growth retardants on the performance of triploid crossandra (Crossandra undulaefolia). South Indian Horticulture., 52: 216-221.

Vimala R, M. Suriachandraselvan.2009. Induced resistance in bhendi against powdery mildew by foliar application of salicylic acid. J. Biopesticides., 2: 111114.

Young Cheol kim, Hyunchae Jung and Kil young kim. 2008. An effective biocontrol bioformulation against Phytophthora blight of pepper using growth mixtures of combined chitinolytic bacteria under different field conditions. European Journal of Plant Pathology, 120: 373-382.

\section{How to cite this article:}

Mallaiah, B., M. Muthamilan and Bhadru, D. 2020. Pot Culture Studies on Integrated Effect of Bio agents, Organic Amendments, Nanoparticles and Chemicals on Crossandra (Crossandra infundibuliformis L. Nees) Wilt Incited by Fusarium incarnatum (Desm.) Sacc. Int.J.Curr.Microbiol.App.Sci. 9(08): 3986-3995. doi: https://doi.org/10.20546/ijcmas.2020.908.459 